\title{
Article
}

\section{Posttranslational Modifications of Microfibril Associated Glycoprotein-1 (MAGP-1)}

Barbara Crippes Trask, Thomas Broekelmann, Timothy M. Ritty, Timothy M. Trask, Clarina Tisdale, and Robert P. Mecham

Biochemistry, 2001, 40 (14), 4372-4380• DOI: 10.1021/bi002738z • Publication Date (Web): 15 March 2001

Downloaded from http://pubs.acs.org on February 21, 2009

\section{More About This Article}

Additional resources and features associated with this article are available within the HTML version:

- $\quad$ Supporting Information

- Access to high resolution figures

- $\quad$ Links to articles and content related to this article

- $\quad$ Copyright permission to reproduce figures and/or text from this article

\section{View the Full Text HTML}




\title{
Posttranslational Modifications of Microfibril Associated Glycoprotein-1 $(\text { MAGP-1) })^{\dagger}$
}

\author{
Barbara Crippes Trask, Thomas Broekelmann, Timothy M. Ritty, Timothy M. Trask, Clarina Tisdale, and \\ Robert P. Mecham* \\ Department of Cell Biology and Physiology, Washington University School of Medicine, St. Louis, Missouri 63110
}

Received November 30, 2000; Revised Manuscript Received February 6, 2001

\begin{abstract}
Microfibril-associated glycoprotein-1 (MAGP-1) is a small molecular weight protein associated with extracellular matrix microfibrils. Biochemical studies have shown that MAGP-1 undergoes several posttranslational modifications that may influence its associations with other microfibrillar components. To identify the sites in the molecule where posttranslational modifications occur, we expressed MAGP-1 constructs containing various point mutations as well as front and back half truncations in $\mathrm{CHO}$ cells. Characterization of transiently expressed protein showed that MAGP-1 undergoes O-linked glycosylation and tyrosine sulfation at sites in its amino-terminal half. This region of the protein also served as a major amine acceptor site for transglutaminase and mediated self-assembly into high molecular weight multimers through a glutamine-rich sequence. Fine mapping of the modification sites through mutational analysis demonstrated that Gln20 is a major amine acceptor site for the transglutaminase reaction and confirmed that a canonical tyrosine sulfation consensus sequence is the site of MAGP-1 sulfation. Our results also show that O-glycosylation occurs at more than one site in the molecule.
\end{abstract}

The formation of a functional elastic fiber requires the processing of tropoelastin monomers into a cross-linked polymer. Ultrastructural studies of elastic fiber assembly in developing tissues have suggested that $10-12 \mathrm{~nm}$ beaded filaments called microfibrils provide the alignment scaffold for elastin cross-linking at sites of fiber assembly (1). Much of what is known about microfibrillar composition has been ascertained through experiments using protein extracted from fetal or neonatal elastic tissues. Functional studies with these tissue extracts, however, have yielded little information because the isolation of monomeric microfibrillar proteins requires denaturation with chaotropic agents and the reduction of disulfide bonds. Furthermore, nonreducible crosslinks acting to stabilize preexisting interactions form as the tissue ages, making extraction and isolation of microfibrillar proteins particularly difficult from adult tissues. These crosslinks have been demonstrated to result, in part, from the action of tissue transglutaminase $(2,3)$.

It is clear from biochemical studies that fibrillins- 1 and -2 play critical structural roles in microfibrillar architecture $(4,5)$. These large, modular glycoproteins are highly homologous, consisting of repeating calcium-binding epidermal growth factor-like domains interspersed between unique 8-cysteine domains. The fibrillins are thought to create the core structure of microfibrils through a head-totail alignment of monomer bundles (6). Ultrastructurally,

$\doteqdot$ This work was supported by grants from the National Institutes of Health HL53325, HL29594, and HL41926 to R.P.M.

* To whom correspondence should be addressed: Department of Cell Biology and Physiology, Washington University School of Medicine, Box 8228, 660 South Euclid Ave., St. Louis, MO 63110. Tel: 314-362-2254. Fax: 314-362-2252. E-mail: bmecham@ cellbiology.wustl.edu. microfibrils are characterized by a "beads on a string" appearance, with fibrillin molecules contributing to both the bead and interbead regions. The composition of the bead, however, is less clearly defined.

Microfibril-associated glycoprotein-1 (MAGP-1) ${ }^{1}$ and its structural relative MAGP-2 (7) are small molecular weight proteins that have been localized to fibrillin-containing microfibrils (8). MAGP-1 is associated with all microfibrils with only two exceptions: those directly adjacent to the plasma membrane of aortic endothelial cells and those at the junction of the zonule and the lens capsule of the eye $(8-10)$. Although MAGP-2 is specifically associated with elastin-associated microfibrils and elastin-free microfibrils in a number of tissues, the protein exhibits patterns of tissue localization and developmental expression that are more restricted than those of MAGP-1 (11). The association of MAGP-1 and -2 with other microfibrillar proteins is apparently covalent since reducing agents are required for their extraction.

It is not yet known what role MAGP-1 plays in microfibrillar structure or function. Previous studies have demonstrated that it is capable of binding tropoelastin, an interaction that may facilitate the linking of elastin monomers onto microfibrils for their proper alignment. The aminoterminal half of MAGP-1 is enriched in acidic amino acids resulting in a cluster of negative charge that has been suggested to interact with a positively charged pocket near the $\mathrm{C}$-terminus of the tropoelastin monomer. Anti-peptide

${ }^{1}$ Abbreviations: DMEM, Dulbecco's modified Eagle's medium; GMEM, Glasgow's modified Eagle's medium; FBC, fetal bovine chondroblasts; IPTG, isopropylthio- $\beta$-galactoside; MAGP, microfibril associated glycoprotein; MSX, methionine sulfoxamine; SDS, sodium dodecyl sulfate. 
antibodies directed against a domain near the $\mathrm{N}$-terminus of MAGP-1 were able to block the incorporation of tropoelastin into fibers by cultured chondrocytes (12). More recently, Finnis and Gibson (13) have localized tropoelastin binding, as well as binding to type VI collagen, to a tyrosine-rich sequence near the amino-terminus of MAGP-1. This region is immediately upstream of that suggested by the antibody studies of Brown-Augsburger et al. (12).

MAGP-1 has been shown to undergo several posttranslational modifications, including glycosylation, sulfation, and transglutamination $(2,14)$. To better characterize these modifications with the aim of assessing their contribution to elastic matrix assembly and function, full-length MAGP-1 and two truncated forms of the protein were stabily expressed in $\mathrm{CHO}$ cells. We show that MAGP-1 undergoes transglutamination, O-linked glycosylation, and tyrosine sulfation at sites in the amino-terminal half of the protein. Precise mapping of these sites using mutational analysis identified Gln20 as the major amine acceptor site for transglutaminase and confirmed that sulfation occurs within a tyrosine sulfation consensus sequence. We also show that at least two sites for glycosylation exist in the amino terminal half of the protein and that a glutamine-rich sequence mediates higher order aggregation.

\section{MATERIALS AND METHODS}

All reagents were obtained from Sigma Chemical Company unless otherwise specified. All restriction enzymes were purchased from Boehringer-Mannheim. Fetal bovine chondrocytes (FBCs) were isolated and maintained as previously described (15). All cloning followed protocols described by Sambrook et al. (16).

Mammalian Expression. Full-length bovine MAGP-1 cDNA (17) was ligated into the pEE 14 mammalian expression vector (18) at the EcoR I site. DH-10b bacteria were transformed with ligated DNA, and subclones containing MAGP-1 cDNA were identified by restriction digestion and confirmed by DNA sequencing. In this vector, expression of the inserted protein is driven by the strong CMV promoter/ enhancer. A glutamine synthase mini-gene, whose expression is driven by the SV-40 promoter, is employed in selection of stable transfectants with MSX.

The MAGP-1-containing mammalian expression plasmid was transfected into $\mathrm{CHO}$ cells using Lipofectin (Gibco/BRL) according to the manufacturer's instructions. After 2 days, transfected cells were split 1:3 into GMEM without glutamine (Gibco/BRL) to which 10\% dialyzed fetal calf serum (FCS) and $25 \mu \mathrm{M}$ MSX had been added. After $\sim 15$ days, colonies were selected and screened for MAGP-1 production by Western blotting of conditioned medium. Positive colonies were cloned by serial dilution, and subclones were screened for MAGP-1 production by Western blotting.

Truncated MAGP-1 constructs encoding the front and back halves of the protein were generated by PCR cloning using full-length cDNA in pBlueScript (pBS, Promega) as a template and oligonucleotide primers containing engineered restriction sites. Amplification with Klentaq DNA polymerase (obtained from Dr. Wayne Barnes, Washington University) was done using a hot start followed by 30 cycles of 2 min at $97{ }^{\circ} \mathrm{C} ; 2$ min at $55^{\circ} \mathrm{C}$; and $3 \mathrm{~min}$ at $72{ }^{\circ} \mathrm{C}$. The amino-terminal half of MAGP-1 was constructed using a $5^{\prime}$ primer complementary to the T3 sequence of the cloning vector (5'-ATTAACCCTCACTAAAG-3'). The 3' primer was reverse complementary to bases $283-306$ of the bovine sequence (5'-GGTCCCGGCTCCCCGGGCTCCGTC-3') with bases 293_G and 294_G to generate a Sma I restriction site near the $3^{\prime}$ end. The 395-bp PCR product was treated with the Klenow fragment of DNA polymerase 1 (Gibco/BRL), gel purified, and digested with Xba I and Sma I. This fragment was ligated into the pEE 14 vector cut with $X b a$ I and Sma I. Ligation into these sites resulted in the construct MAGP-1 (M1-P89) with the addition of six amino acid residues at the carboxyl-terminal end (-Gly-Arg-AlaArg-Ile-His) before the vector-derived stop codon was reached.

To generate the carboxy-terminal half of MAGP-1, bases 287-936 (includes some 3' UTR) were PCR amplified using Klentaq DNA polymerase. Full-length bovine cDNA in pBS was used as a template. A $5^{\prime}$ primer complementary to bases 286-304 of the bovine sequence with $298 \Delta \mathrm{T}$ generated a BamH I site near the $5^{\prime}$ end (5'-GAGCCCACGGATCCGGGA-3'). The 3' primer used was complementary to the $\mathrm{T} 7$ sequence in pBS. The $725 \mathrm{bp}$ PCR product was treated as above before digestion with BamH I and Hind III and ligation into pBS digested with the same enzymes. The MAGP-1 leader sequence was PCR amplified with Taq DNA polymerase from the full-length cDNA template. The same T3 $5^{\prime}$ primer described above and a $3^{\prime}$ primer reverse complementary to bases $80-103$ of the bovine sequence (5'CAGGGGGTCCTGATCATACTGGCC-3') with base $93 \Delta$ A were used to generate a $\mathrm{Bcl}$ I restriction site at the $3^{\prime}$ end. This 190-bp PCR product was treated as above before digestion with $\mathrm{Xba}$ I and $\mathrm{Bcl} \mathrm{I}$ and was ligated into pBS containing the amino-terminal truncated construct cut with $X b a$ I and BamH I. These steps generated a product consisting of the MAGP-1 leader sequence plus the first four amino acids of the mature protein fused to amino acids E91C183. This 915-bp construct was digested with $\mathrm{Xba}$ I and EcoR V and ligated into the pEE 14 vector cut with the same enzymes. Both truncated proteins were transfected into $\mathrm{CHO}$ cells and stable transfectants were selected as described above for the full-length protein.

Point mutations for mammalian expression were made using the QuikChange, (Stratagene) site-directed mutagenesis kit per the manufacturer's specifications with full-length MAGP-1 in pBS as the template. Briefly, this protocol uses $P f u$ DNA polymerase along with complimentary forward and reverse primers containing the desired mutation(s) to amplify around a circular template. Following amplification, the methylated, wild-type template (plasmid DNA from E. coli) is degraded with $D p n$ I while the unmethylated PCR product containing the incorporated mutations remains intact. This PCR-derived plasmid containing the mutagenized sequence is transformed into E. coli and manipulated as desired. Three threonine residues (T54, T79, and T90) were mutated to valine using the following primers: bases $175-196$ with $185 \Delta \mathrm{G}$ and $186 \Delta \mathrm{T}\left(5^{\prime}\right.$-CCCAGAGATGGTCCCTCGGCCG$3^{\prime}$ ) for T54 $4 \mathrm{Val}$; bases $251-271$ with $260 \Delta \mathrm{G}$ and $261 \Delta \mathrm{T}$ (5'-CCTGCCCCCGTCTTAGAACCG-3') for T79 $\Delta \mathrm{Val}$; and bases 285-306 with $293 \Delta \mathrm{G}, 294 \Delta \mathrm{T}$, and $295 \Delta \mathrm{C}$ (5'CGGAGCCCGTCGAGCCGGGACC-3') for T90 $\Delta$ V. To generate mutation in the tyrosine sulfation consensus sequence, primers complimentary to bases $151-188$ of the 
bovine sequence with $165 \Delta \mathrm{C}, 168 \Delta \mathrm{C}$, and $174 \Delta \mathrm{C}$ (5'-CGAGAATCCGGACTCCTCTGACTCCCCAGAGATGACCC-3') were used to PCR amplify the MAGP-1 mutant sequence yielding a construct with Y47, Y48, and Y50 all changed to serine residues. In all cases, products were sequenced to confirm incorporation of the desired mutation and to ensure that no additional mutations were introduced. The mutant MAGP-1 sequences were cut out of pBS with $X b a$ I and Hinc II and ligated into the pEE 14 mammalian expression vector cut with Xba I and Sma I. All constructs were transiently transfected into $\mathrm{CHO}$ cells along with wild-type MAGP-1 as described for stable transfections above.

Antibodies. Bovine MAGP-1 cDNA was digested with Pst I and $A p a \mathrm{~L}$ I, yielding a 270-nt fragment that was filled in with the large fragment of DNA Polymerase and re-ligated into Pst I and EcoR V-cut pBS. This construct was digested with BamH I and Xho I, and the insert was ligated into the pGEX vector (Pharmacia), resulting in a fusion protein of glutathione $S$-transferase (GST) coupled with amino acids G14-P103 of MAGP-1. E. coli containing this plasmid were grown in an environmental shaker at $37^{\circ} \mathrm{C}$ to an O.D. of 0.4 before induction of fusion-protein synthesis with $0.2 \mathrm{mM}$ IPTG (Gibco/BRL) for $4 \mathrm{~h}$. Bacteria were pelleted and subsequently lysed by repeated freeze-thaw cycles followed by addition of $0.1 \%$ Triton X-100 and sonication. Bacterial lysate was cleared of debris by centrifugation. The supernatant was then absorbed to a glutathione-Sepharose 4B column (Pharmacia) and bound fusion protein was eluted with PBS containing $10 \mathrm{mM}$ glutathione.

The carboxy-terminal construct of MAGP-1 described above for mammalian expression (lacking the leader sequence) was ligated into the bacterial expression vector $\mathrm{pQE}$ (Qiagen) at the BamH I and Hind III sites. This expression vector contains an $\mathrm{N}$-terminal $6 \mathrm{XHis}$ tag that was used for purification of the recombinant protein. Subclones containing the appropriate recombinant plasmid were identified by restriction digestion. DNA was transformed into M15 bacteria and clones were screened for protein production. One positive clone was selected and was grown for $4 \mathrm{~h}$ at $37^{\circ} \mathrm{C}$, induced with $1 \mathrm{mM}$ IPTG for $4 \mathrm{~h}$ at $37^{\circ} \mathrm{C}$, pelleted, and lysed with $6 \mathrm{M}$ urea, $\mathrm{pH} 8$, overnight at $4{ }^{\circ} \mathrm{C}$. Bacterial lysate was cleared by centrifugation, and the supernatant was mixed with nickel agarose (Qiagen). After extensive washing at $\mathrm{pH} 8$ and $\mathrm{pH} 6$, bound protein was eluted with $6 \mathrm{M}$ urea, $\mathrm{pH} 5$.

Antibodies were generated by injection of purified recombinant protein into rabbits. Serum was collected and antibodies were purified over an affinity column containing elastic fiber proteins extracted from bovine nuchal ligament coupled to Sepharose (14). Anti-peptide antibodies generated to peptide 244 (amino acids G19-D31), peptide 243 (amino acids Y38-E52), and peptide 239 (amino acids V74-T87) of bovine MAGP-1 were previously described by BrownAugsburger et al. (12).

Western Blotting. Serum-free GMEM was conditioned overnight by MAGP-1-expressing CHO cells. The conditioned medium was then concentrated and subjected to reducing SDS-PAGE with transfer of protein to nitrocellulose. Filters were blocked in PBS/3\% nonfat dry milk for $1 \mathrm{~h}$ at room temperature and incubated with primary antibodies, each at a 1:1000 dilution, overnight at $4{ }^{\circ} \mathrm{C}$ in $\mathrm{PBS} / 3 \%$ dry milk. The filters were then washed three times with PBS/milk and incubated with an HRP-conjugated secondary antibody (Amersham) for $1 \mathrm{~h}$ at room temperature in PBS/milk. Filters were washed three times and immunoreactive proteins were visualized by enhanced chemiluminescence.

Immunoprecipitation. Confluent cells were incubated for $5 \mathrm{~h}$ with $50 \mu \mathrm{Ci} / \mathrm{mL}\left[{ }^{35} \mathrm{~S}\right]$-cysteine (sp. act. $>800 \mathrm{Ci} / \mathrm{mmol}$; $\mathrm{ICN}$ ) in cysteine-free DMEM, $42 \mu \mathrm{Ci} / \mathrm{mL}\left[{ }^{14} \mathrm{C}\right]$-proline (sp. act. $>225 \mathrm{mCi} / \mathrm{mmol}$; ICN) in DMEM, or $150 \mu \mathrm{Ci} \mathrm{Na}\left[{ }^{35} \mathrm{~S}_{\mathrm{O}} \mathrm{O}_{4}\right.$ (sp. act. $\sim 43 \mathrm{Ci} / \mathrm{mg} \mathrm{S} ; \mathrm{ICN}$ ) in sulfate-free S-MEM (Gibco/ BRL). To assess sugar incorporation, confluent MAGP-1expressing $\mathrm{CHO}$ cells were labeled overnight in $5 \mathrm{~mL} / \mathrm{p} 100$ tissue culture dish with $50 \mu \mathrm{Ci} / \mathrm{mL}\left[{ }^{3} \mathrm{H}\right]$-glucosamine (sp. act. 25-40Ci/mmol; ICN) in low-glucose DMEM (Gibco/ BRL) containing $2 \%$ dialyzed FCS. CHO cells transiently transfected with wild type and point mutation-containing constructs were radiolabeled as above 4 days after transfection. For all immunoprecipitations, radiolabeled medium was pre-cleared with Protein-A immobilized on Trysacryl (Pierce) for $1 \mathrm{~h}$ at room temperature. Beads were pelleted, and the supernatant was transferred to a new tube containing antibody and Protein-A/Trysacryl and incubated at $4{ }^{\circ} \mathrm{C}$ overnight. Beads were pelleted and washed $4 \times$ with detergent buffer containing $0.1 \%$ SDS, $0.25 \%$ deoxycholate, $2 \mathrm{mM}$ EDTA, $0.5 \%$ Triton $\mathrm{X}-100,150 \mathrm{mM} \mathrm{NaCl}$, and $50 \mathrm{mM}$ Tris, $\mathrm{pH} 8$. Washed beads were then resuspended in reducing sample buffer before electrophoresis.

In Vitro Transglutaminase Assay. To generate a recombinant protein containing the putative N-terminal transglutaminase site, the MAGP-1 (G14-P103)/pGEX construct described above for generation of an MAGP-1 antibody was digested with Eag I. This digestion liberated a fragment consisting of the GST moiety fused to amino acids G14P27 of MAGP-1 that was then re-ligated into the pGEX vector. Recombinant protein generated from this construct was purified in the same manner as the larger fusion protein described above. Both of these bacterial fusion proteins were incubated in a $50 \mathrm{mM}$ Tris, $\mathrm{pH} 8.3$, buffer containing 100 $\mu \mathrm{M} \mathrm{CaCl}_{2}$ with 1.2 units of guinea pig liver transglutaminase and $0.65 \mu \mathrm{Ci}\left[{ }^{14} \mathrm{C}\right]$-spermidine (Amersham) in a final reaction volume of $45 \mu \mathrm{L}$. Reactions were allowed to proceed at 37 ${ }^{\circ} \mathrm{C}$ for $1.5 \mathrm{~h}$. Reactions were stopped by the addition of 2 $\mu \mathrm{L}$ of $0.5 \mathrm{M}$ EDTA. Incorporation of radiolabeled spermidine was detected by electrophoresis of the reaction products on $10 \%$ SDS-PAGE gels followed by autoradiography.

Point mutations of the glutamine residues within the shorter construct were made using the QuikChange system as described above for mutations in proteins for mammalian expression. The MAGP-1 (G14-P57) construct in pGEXserved as a template in generating these mutant fusion proteins. Three of the four glutamine residues contained within this construct, Q20, Q34, and Q41, were mutated independently to Asn by PCR using the following primers: bases $73-98$ with $82 \Delta \mathrm{A}$ and $84 \Delta \mathrm{T}$ (5'-GGCTCAGGGCAATTATGATCTGGACC-3') for Q20 $\Delta \mathrm{N}$; bases 112-137 with $125 \Delta \mathrm{A}$ and $127 \Delta \mathrm{C}$ (5-CCCAGACCACGTGAACTATACCCACT-3') for Q34 $\Delta \mathrm{N}$; and bases $139-161$ with $146 \Delta \mathrm{A}$ and $148 \Delta \mathrm{T}$ (5'-CAGCGAGAATATCGAGAATCCGG-3') for $\mathrm{Q} 41 \Delta \mathrm{N}$. These recombinant mutant proteins were isolated by glutathione affinity chromatography, and transglutaminase assays were performed as described above for the wild-type proteins. 
Peptide Aggregation and Congo Red Assay. Peptide 127 encoding amino acids 57-80 of MAGP-1 (PPEEQFQFQSQQQVQQEVIPAPTL) was synthesized using FAST-Moc chemistry and dissolved at $10 \mathrm{mg} / \mathrm{mL}$ in $7 \mathrm{M}$ guanidine $\mathrm{HCl}$. A total of $10 \mu \mathrm{L}$ of the peptide stock solution was diluted with $200 \mu \mathrm{L}$ of PBS and $200 \mu \mathrm{L}$ of water and subjected to slow rotation overnight at room temperature. Precipitated peptide was pelleted by centrifugation in a microfuge, smeared on a glass slide, fixed in $95 \%$ ethanol, and stained with $0.05 \%$ congo red in $50 \%$ glycerol. Evaluation of congo red staining by polarization microscopy was performed using a Zeiss Axioskop equipped with optimally aligned crosspolarizers.

Digestion of Carbohydrate Chains and Lectin Binding. For removal of carbohydrates, Trisacryl beads with immunoprecipitated protein were resuspended in $25 \mu \mathrm{L}$ of $10 \mathrm{mM}$ calcium acetate/20 mM sodium cacodylate, $\mathrm{pH}$ 7.4. Neuraminidase $(25 \mathrm{mU})$ was then added, and samples were incubated for $1 \mathrm{~h}$ at $37{ }^{\circ} \mathrm{C}$ followed by an overnight incubation with 4 mU O-glycanase (Genzyme). Reactions were stopped by the addition of SDS-PAGE reducing sample buffer. Following electrophoresis, gels were fixed, treated with $\mathrm{En}^{3} \mathrm{Hance}$ (Amersham), and dried before visualization of immunoprecipitated proteins by autoradiography.

Untransfected CHO cells or CHO cells expressing recombinant protein were incubated overnight in serum-free medium. The conditioned medium was then incubated for 1 $\mathrm{h}$ at $37^{\circ} \mathrm{C}$ with 1 unit/mL neuraminidase to remove terminal sialic acid residues from glycoproteins. Treated samples were rotated end-over-end at room temperature with uncoupled agarose beads for $1 \mathrm{~h}$ to decrease nonspecific protein association with agarose. The beads were pelleted and the supernatants were transferred to new tubes containing washed control agarose or Jacalin-agarose beads (Vector Labs). Samples were allowed to rotate end-over-end at $4{ }^{\circ} \mathrm{C}$ overnight. Beads were pelleted by centrifugation and washed four times with serum-free GMEM. Washed beads were resuspended in reducing sample buffer and analyzed by SDS-PAGE followed by Western blotting.

Tropoelastin Binding Assay. MaxiSorp (Nunc) plates were coated with $1 \mu \mathrm{g} /$ well of recombinant human tropoelastin (kind gift of Dr. Joel Rosenbloom, University of Pennsylvania) in $10 \mathrm{mM}$ bicarbonate buffer, $\mathrm{pH} 10$, in a humidified chamber at $4{ }^{\circ} \mathrm{C}$ overnight. Following coating, plates were washed $3 \times$ with PBS and blocked with $1 \%$ BSA in PBS for $1 \mathrm{~h}$ at $37{ }^{\circ} \mathrm{C}$ and again washed $3 \times$ with PBS. Medium conditioned overnight by MAGP-1-expressing $\mathrm{CHO}$ cells was used as a source for MAGP-1 protein. The same cells treated with $10 \mathrm{mM} \mathrm{KClO}_{3}$ were used as a source for nonsulfated MAGP-1. Medium from treated and untreated cells was diluted 1:1 (v/v) with $1 \%$ BSA in PBS. Subsequent serial dilutions were made in PBS containing $0.1 \%$ BSA. The diluted medium samples in $100 \mu \mathrm{L}$ volumes were added to the tropoelastin-containing plates and incubated for 90 min at $37{ }^{\circ} \mathrm{C}$ in a $5 \% \mathrm{CO}_{2}$ incubator. Unbound protein was removed with three washes of PBS. Bound protein was detected using polyclonal anti-GST-MAGP-1 antibody followed by an HRP-coupled anti-rabbit IgG secondary antibody (BioRad) and color development with ABTS (Kirkegaard). Antibodies were diluted in PBS containing 0.1\% BSA and incubations were done for $1 \mathrm{~h}$ at $37{ }^{\circ} \mathrm{C}$. The color reaction was quantified using a Bio-Rad microtiter plate
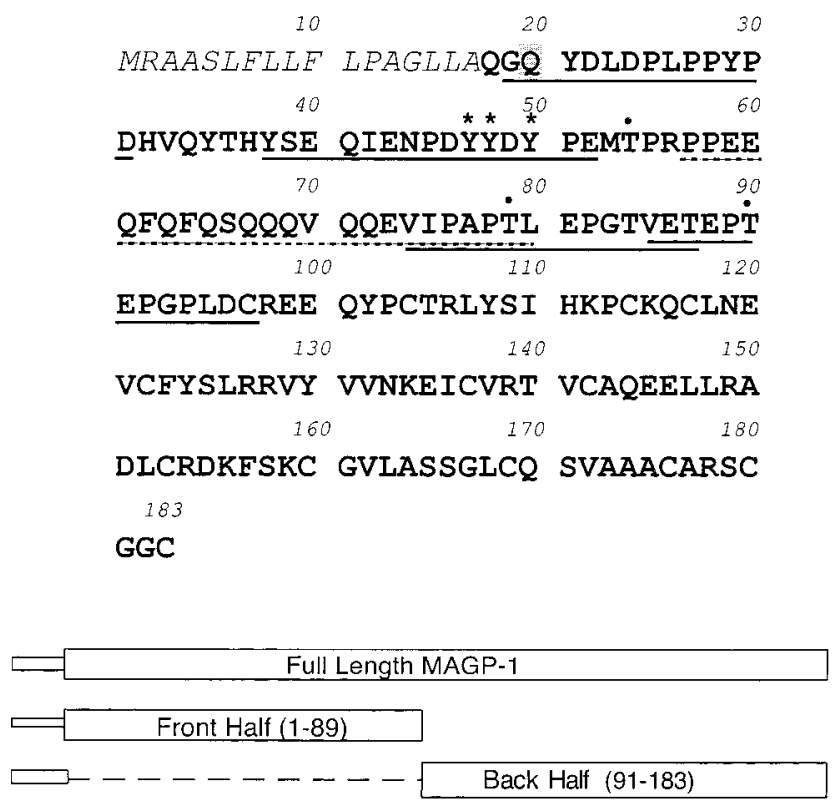

FIGURE 1: Sequence of full-length bovine MAGP-1 and schematic representation of the constructs used in this study. (Top) full-length sequence of bovine MAGP-1 indicating peptide sequences for synthetic peptides (underlined) and sites of posttranslational modification determined in this study. Peptide P57-L80 (dashed underline) contains the glutamine-enriched region of the protein. Threonine residues predicted as sites of glycosylation are indicated by (). Sites of transglutamination are shaded. Tyrosine residues in the sulfation consensus site are marked by an asterisk. The leader sequence is in italics. (Bottom) Schematic representation of the MAGP-1 constructs stably expressed in CHO cells.

reader at $405 \mathrm{~nm}$.

\section{RESULTS}

Expression of Full-Length and Truncated MAGP-1 Proteins in CHO Cells. To characterize sites of posttranslational modifications in MAGP-1, we expressed full-length, back and front half constructs (Figure 1) of the bovine protein in $\mathrm{CHO} \mathrm{K} 1$ cells. These cells were chosen because of their ease of transfection and the absence of detectable endogenous MAGP-1 expression. The front and back half constructs were designed to encode the two discreet domains of this protein that are thought to exhibit distinct functions within the elastic matrix $(2,17)$.

Figure 2 shows that each of the recombinant proteins is expressed by transfected cells and that the antibodies used in this study are specific for their respective antigens and do not react with $\mathrm{CHO}$ proteins expressed by untransfected cells. Expression of the amino-terminal half of MAGP-1 results in the secretion of multiple aggregate forms of the protein (Figure 2, panel A). Because this construct contains none of the protein's cysteine residues, the aggregation is not due to disulfide bonding. It does, however, appear to be an irreversible interaction as we have been unable to either inhibit or reverse aggregate formation by the addition of 8 $\mathrm{M}$ urea (data not shown). Like the front half construct, the back half fragment was secreted into the medium at high levels but showed no aggregation (Figure 2, panel B).

Sequences responsible for aggregation of the front half of the molecule were determined by testing the solubility of synthetic peptides that encode most of the front half construct. Of the five peptides tested (G19-D31, Y38-E52, 


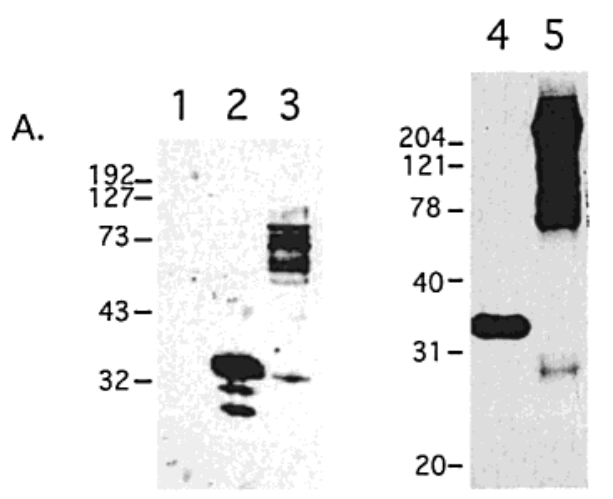

Front Half (amino acids 1-89)

B.

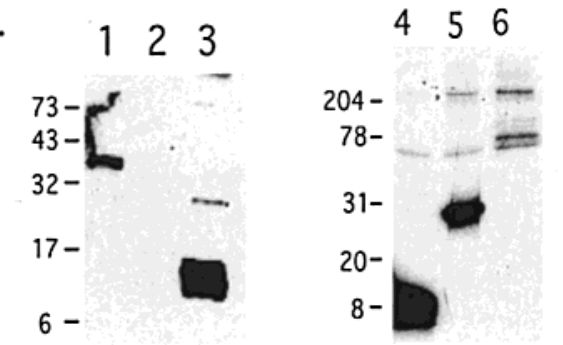

Back Half (amino acids 91-183)

FIGURE 2: Expression of full-length MAGP-1 and truncation constructs in $\mathrm{CHO}$ cells (A) Immunoblots of conditioned medium from untransfected CHO K1 (lane 1), CHO cells stably transfected with full-length MAGP-1 (lane 2), and the front half of MAGP-1 (lane 3) developed with an antibody generated to the MAGP-1/ GST fusion protein. A similar pattern was obtained when the antibody was used for immunoprecipitation of the full-length (lane 4) and front half (lane 5) constructs from culture medium of $\left[{ }^{14} \mathrm{C}\right]-$ proline-labeled cells. (B) Detection of the back half construct using an antibody generated to a bacterial fusion protein encoding the carboxy-terminal half of MAGP-1. Lane 1 shows full-length MAGP-1 and lane 3 the carboxy-terminal half of the protein detected by immunoblot. No immunoreactive protein was detected in the medium of untransfected CHO cells (lane 2). Lanes 4 and 5 show immunoprecipitation products of $\left[{ }^{14} \mathrm{C}\right]$-proline-labeled $\mathrm{CHO}$ cells expressing the back half and full-length constructs, respectively. No $\mathrm{CHO}$ proteins in these molecular weight ranges were immunoprecipitated from untransfected cells (lane 6). Gels were run under reducing conditions. Molecular weight markers are in kilodaltons.

P57-L80, V74-T87, and V85-C97; see Figure 1), the only peptide found to be insoluble under physiological conditions was P57-L80. This peptide contains the glutamine-rich sequence indicated by a dashed underline in Figure 1. Although soluble in $8 \mathrm{M}$ guanidine $\mathrm{HCl}$, the peptide precipitated in the form of small uniform fibers when the guanidine/peptide solution was diluted with water. The peptide precipitates showed green birefringence under polarized light following staining with congo red (data not shown), suggestive of a $\beta$-pleated sheet conformation (19).

Identification of Transglutamination Site in MAGP-1. Previous studies have suggested that an amine acceptor site for the transglutaminase reaction is located near the amino terminus of MAGP-1 (2). To better localize the site on MAGP-1 that is modified by this enzyme, two overlapping GST bacterial fusion proteins were made. The longer of the two constructs (amino acids G14-P103) contains 13 of the

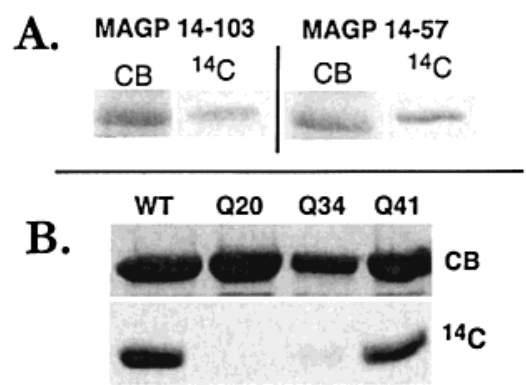

FIGURE 3: Transglutaminase site is localized to Gln20. (A) MAGP-1 constructs encoding amino acids G14-P103 and G14P57 were expressed as GST fusion proteins and incubated with $\left[{ }^{14} \mathrm{C}\right]$-spermidine in the presence of transglutaminase. $\mathrm{CB}$, Coomassie blue stains of protein purified using a glutathione-Sepharose column; ${ }^{14} \mathrm{C}$, incorporation of $\left[{ }^{14} \mathrm{C}\right]$-spermidine. (B) Recombinantly expressed proteins with individual point mutations. WT, unmutated protein; Q20, Gln20 to Asn mutation; Q34, Gln34 to Asn mutation; $\mathrm{Q} 41$, Gln41 to Asn mutation. CB, Coomassie blue stain; ${ }^{14} \mathrm{C}$, incorporation of $\left[{ }^{14} \mathrm{C}\right]$-spermidine.

16 glutamine residues found in the full-length protein. The smaller fusion protein (amino acids G14-P57), which contained the putative acceptor site, was generated by taking advantage of a convenient restriction site in the longer construct. This polypeptide contains the first four glutamine residues in the amino-terminal region of MAGP-1. Figure 3 , panel A shows that both the shorter (G14-P57) and longer (G14-P103) proteins incorporate $\left[{ }^{14} \mathrm{C}\right]$-spermidine when incubated with transglutaminase. It was necessary to include the GST moiety on peptides containing the glutamine-rich sequence to prevent aggregation. GST alone was not a substrate for transglutaminase (not shown).

To identify the specific residues acting as amine acceptors, three of the four glutamines in the shorter construct were individually mutated to asparagine residues. The glutamine at the amino-terminus of secreted mammalian MAGP-1 (Q18) forms pyroglutamic acid (2) and therefore cannot act as an acceptor residue. Figure 3, panel B, shows that two of the mutant recombinant proteins, Q34N and Q41N, continue to serve as substrates for transglutaminase, although the level of labeling of the Q34N mutant is minimal as compared to the wild type or Q41N proteins. Mutation of Q20 completely blocked incorporation of $\left[{ }^{14} \mathrm{C}\right]$ label, indicating that this residue is the major site for transglutamination in this region of the protein.

MAGP-1 Contains O-Linked Carbohydrate(s). Uncertainty as to whether MAGP-1 is a glycoprotein was raised when no N-linked glycosylation sites were found in the protein's primary sequence (17). To determine whether the protein contains O-linked sugars, we incubated $\mathrm{CHO}$ cells expressing full-length MAGP-1 with $\left[{ }^{3} \mathrm{H}\right]$-glucosamine. While glucosamine is not incorporated directly into O-linked carbohydrates, most cells will epimerize this carbohydrate to galactosamine, which is incorporated. Figure 4 shows incorporation of the radiolabeled sugar into full-length MAGP-1 immunoprecipitated from culture medium (lane 1). Removal of the glucosamine label with O-glycanase (lane 2) confirms its incorporation into O-linked carbohydrate chains.

Additional evidence that MAGP-1 contains O-linked carbohydrate was obtained using Jacalin, a lectin that recognizes the galactosyl $(\beta-1,3) N$-acetylgalactosamine structure of O-linked carbohydrates. Serum-free medium 

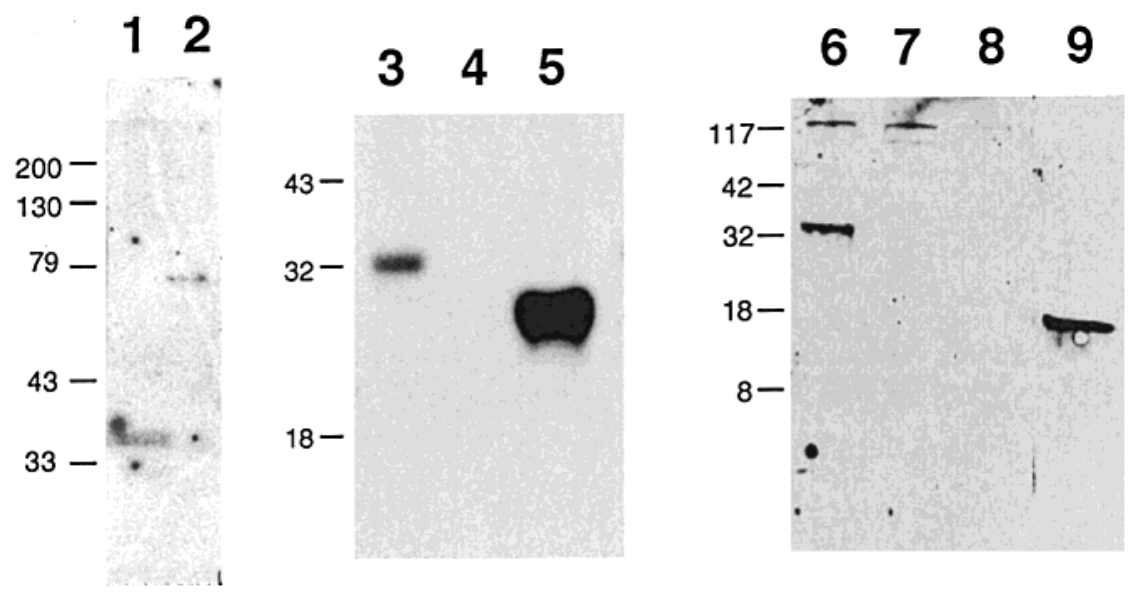

FIGURE 4: MAGP-1 is O-linked glycosylated. Lane 1 shows an immunoprecipitation of MAGP-1 from transfected CHO cells radiolabeled with $\left[{ }^{3} \mathrm{H}\right]$-glucosamine. Treatment of immunoprecipitated protein with neuraminidase and $O$-glycanase removes all carbohydrates associated with MAGP-1 (lane 2). Secreted, sulfate-labeled full-length MAGP-1 binds Jacalin-agarose beads (lane 3), as does the amino-terminal half of the protein (lane 5). No sulfate-labeled proteins secreted by untransfected CHO cells bind to Jacalin (lane 4). Because the back half of MAGP is not sulfated, its binding to the Jacalin lectin was detected by immunoblot using the C-terminal antibody. Lane 6 shows binding of the full-length protein, whereas no binding was detected for the back half construct (lane 8). Lane 7 is medium from untransfected CHO cells. Lane 9 shows isolated back half protein used as a marker to confirm antibody reactivity.

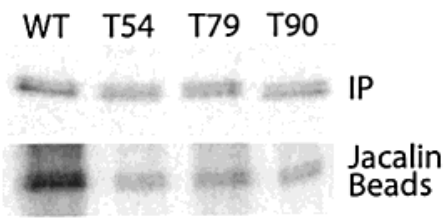

FIGURE 5: Mutation of Thr54, Thr79, or Thr90 suggests multiple glycosylation sites in MAGP-1. (A) Immunoprecipitation (top) from the medium of $\left[{ }^{35} \mathrm{~S}\right]$-cysteine labeled $\mathrm{CHO}$ cells transiently transfected with mutant MAGP-1 proteins demonstrates secretion levels similar to those of wild-type protein. Sulfate-labeled medium was also mixed with Jacalin-agarose beads (bottom) and bound protein assayed by SDS-PAGE. The bottom panel shows that all of the mutant proteins bound to the lectin, suggesting that a single point mutation at any of the three threonine residues targeted in this study is not sufficient to prevent O-linked glycosylation of MAGP-1. WT, wild-type MAGP-1; T54, Thr54 mutant; T79, Thr79 mutant; T90, Thr90 mutant.

conditioned by $\mathrm{CHO}$ cells expressing full-length and truncated MAGP-1 proteins was treated with neuraminidase to remove terminal sialic acid residues and then incubated with either control agarose or Jacalin-coupled agarose. Protein that bound to the lectin was subjected to electrophoresis and MAGP-1 was visualized by Western blotting. Figure 4 shows that the full-length (lane 3) and the amino-terminal half of the protein (lane 5) associate specifically with Jacalin agarose while the carboxy-terminal half (lane 8) does not. This demonstrates that carbohydrate modification of MAGP-1 occurs between residues 1 and 89 of the mature protein.

Submission of the bovine MAGP-1 sequence to the NetOglyc Prediction Server (www.cbs.dtu.dk/service/netOGlyc/) $(20,21)$ resulted in the identification of three possible threonine glycosylation sites within full-length bovine MAGP1: T54, T79, and T90, with the first two of these having the highest glycosylation potential. The same three residues are predicted for glycosylation in human MAGP-1. To determine whether these residues are active sites of glycosylation, all three residues were independently mutated in the full-length protein. Figure 5 shows that all three mutant proteins are secreted when transiently expressed in $\mathrm{CHO}$ cells. Also shown in Figure 5 is binding of all three mutant proteins to Jacalin agarose, indicating that glycosylation is occurring at

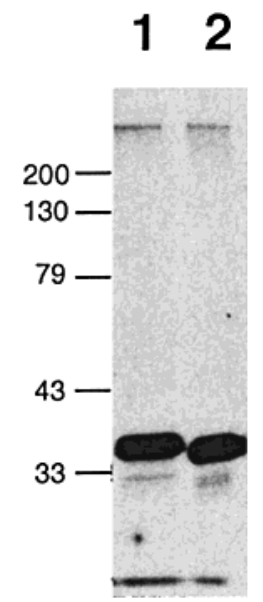

FIGURE 6: Sulfation of MAGP-1 is on the protein, not on carbohydrate. Lane 1 shows an immunoprecipitation of MAGP-1 from transfected $\mathrm{CHO}$ cells radiolabeled with [35S]-sulfate. The presence of the sulfate label after removal of carbohydrates with O-glycanase (lane 2) confirms that the protein, not the carbohydrate, is sulfated.

multiple sites in the protein and that mutation of only one of these amino acids is not sufficient to prevent glycosylation.

MAGP-1 Is Sulfated on Tyrosine Residues. Bovine MAGP-1 has been shown to be sulfated when expressed in SF9 cells (22). To localize the site of sulfation, $\mathrm{CHO}$ cells stably expressing full-length MAGP-1 were incubated in medium containing $\left[{ }^{35} \mathrm{~S}\right]$-sulfate. Figure 6 (lane 1) shows that MAGP-1 immunoprecipitated from the medium of these cells incorporates the sulfate label as expected. Treatment of the immunoprecipitated protein with $O$-glycanase to release carbohydrates (lane 2) did not remove the sulfate radiolabel, demonstrating that the sulfate modification is not on O-linked sugars.

A possible site of modification within the MAGP-1 molecule was suggested by the presence or absence of sulfate labeling in two known degradation products of the protein. The lower molecular weight products (bands B and C indicated by arrows in Figure 7) occur in variable amounts and are routinely seen when MAGP-1 is isolated from tissues 


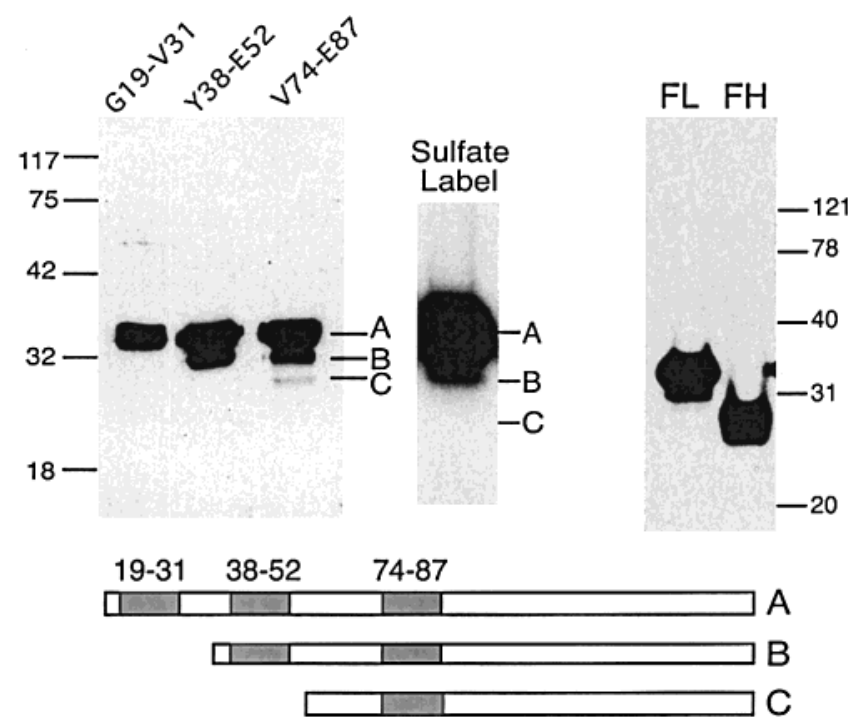

FIGURE 7: The sulfation of MAGP-1 occurs between amino acids 38 and 74. MAGP-1 secreted from CHO cells undergoes partial proteolysis at its amino-terminus. The left panel is an immunoblot of conditioned medium from MAGP-1 CHO cells developed with anti-peptide antibodies that partially map the protease cleavage sites. The most amino-terminal antibody (amino acids 19-33) recognizes only the full-length protein (lane 1). Antibody to amino acids 7486 recognize all three bands (lane 3 ), whereas only the two higher molecular weight bands are recognized by the antibody to amino acids $38-52$ (lane 2 ). Since the $\left[{ }^{35} \mathrm{~S}\right]$-sulfate label is missing from the lower band but is present in the two higher bands (center panel), the sulfate-containing residues must lie between the epitopes recognized by the $38-52$ and $74-86$ antibodies. The right panel shows an immunoprecipitation from $\left[{ }^{35} \mathrm{~S}\right]$-sulfate labeled cells demonstrating that the full-length (FL) and the front-half $(\mathrm{FH})$ constructs, both of which contain the putative sulfation site, are able to incorporate sulfate label.

or cells. Western blotting of MAGP-1 with antibodies raised against three different synthetic peptides (Figure 7, left) demonstrates that bands $\mathrm{B}$ and $\mathrm{C}$ arise as a consequence of amino-terminal deletions. These peptide antibodies also define the missing regions with some accuracy. For example, all three bands react with the antibody raised against amino acids V74-T87, but the antibody raised against amino acids Y38-E52 recognizes only the two higher molecular weight bands. Since the $\left[{ }^{35} \mathrm{~S}\right]$-sulfate label is missing from the lower band but is present in the two higher bands (Figure 7, center), the sulfate-containing residue(s) must lie between the epitopes recognized by the Y38-E52 and V74-T87 antibodies. This is the region bounded by residues 38 and 87 . An analysis of the amino acid sequence defined by this region indicates that tyrosine residues 47,48 , and 50 (asterisks, Figure 1) lie within a consensus sequence for tyrosinesulfation (23). Sulfate incorporation into the amino-terminal construct that contains this sequence, and not the carboxyterminal half (data not shown), indicates the specificity of the sulfation reaction.

To confirm that the tyrosine consensus sequence is in fact the site of MAGP-1 sulfation, all three tyrosine residues within this sequence were mutated to serine. $\mathrm{CHO} \mathrm{K} 1$ cells were transiently transfected with either wild type or mutant protein and immunoprecipitations were performed for secreted MAGP-1. Figure 8 shows that the mutant protein fails to incorporate sulfate label, verifying that the tyrosine consensus sequence is the only site of MAGP-1 sulfation.

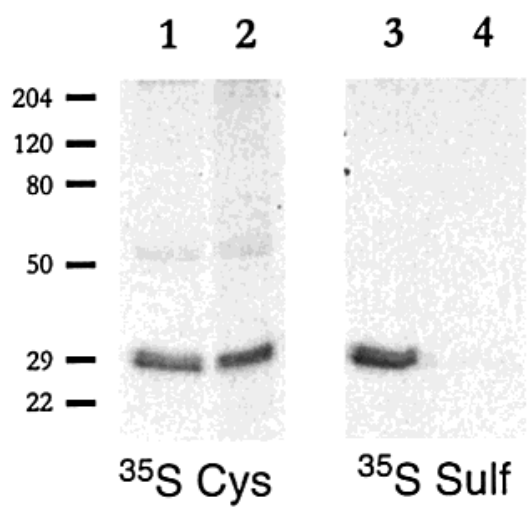

FIGURE 8: The sulfation of MAGP-1 occurs within a tyrosine sulfation consensus sequence. Immunoprecipitation of wild-type MAGP-1 and a mutant protein with Tyr47, Tyr48, and Tyr50 changed to serine residues indicates that the mutated protein fails to incorporate sulfate label. Both the wildtype (lane 1) and the mutated (lane 2) proteins are secreted and incorporate $\left[{ }^{35} \mathrm{~S}\right]$-cysteine. Lanes 3 and 4 demonstrate that the wild-type (lane 3 ) but not the mutated (lane 4) protein is labeled with $\left[{ }^{35} \mathrm{~S}\right]$-sulfate.

Inhibition of Sulfation Does Not Alter MAGP-1's Ability to Bind Tropoelastin. Our previous studies have shown that MAGP-1 binds tropoelastin in solid-phase binding assays. It has been suggested that these two proteins associate via an ionic interaction between the negatively charged aminoterminal region of MAGP-1 and the positively charged C-terminus of tropoelastin (12). Sulfation of the aminoterminal region of MAGP-1 would greatly enhance the negative charge of this region, raising the possibility that its interaction with tropoelastin might depend on this modification.

To test this possibility, medium conditioned by untreated and chlorate-treated cells expressing full-length MAGP-1 was collected and used in a solid-phase tropoelastin binding assay. Chlorate is a potent and specific inhibitor of protein and proteoglycan sulfation (24) but does not generally inhibit protein synthesis. Indeed, neither MAGP-1 synthesis nor its ability to interact with antibody was inhibited by the addition of $10 \mathrm{mM}$ chlorate (Figure 9, panel A, lanes 1 and 4). The relative absence of $\left[{ }^{35} \mathrm{~S}\right]$-sulfate label in MAGP-1 immunoprecipitated from chlorate-treated cells (lane 3) confirms the effectiveness of the treatment. Figure 9, panel B, shows that the absence of tyrosine sulfation does not alter the ability of MAGP-1 to bind tropoelastin, suggesting that sulfation plays little role in the tropoelastin-MAGP-1 interaction.

\section{DISCUSSION}

Several studies have shown that MAGP-1 undergoes posttranslational modifications that may alter its biological properties and influence its interactions with other proteins. The present study focuses on a detailed analysis of the sites in the molecule where these modifications occur.

In earlier studies using MAGP-1 derived from a baculoviral expression system (2), the full length, native protein demonstrated a propensity to aggregate into high molecular weight multimers. When the front and back halves of the molecule were separately expressed in $\mathrm{CHO}$ cells, it was found that aggregation occurred only with the front half of the protein. Analysis of the front half of the molecule using synthetic peptides identified the glutamine-rich motif located between amino acids $61-72$ as the sequence responsible for 


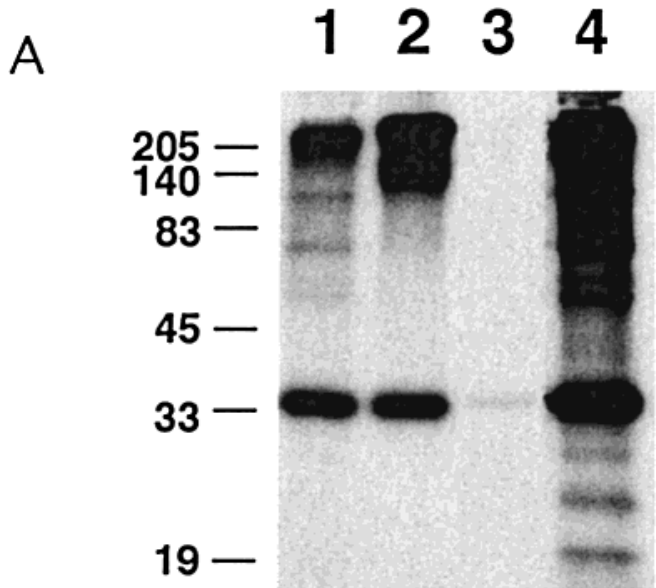

$\mathrm{B}$

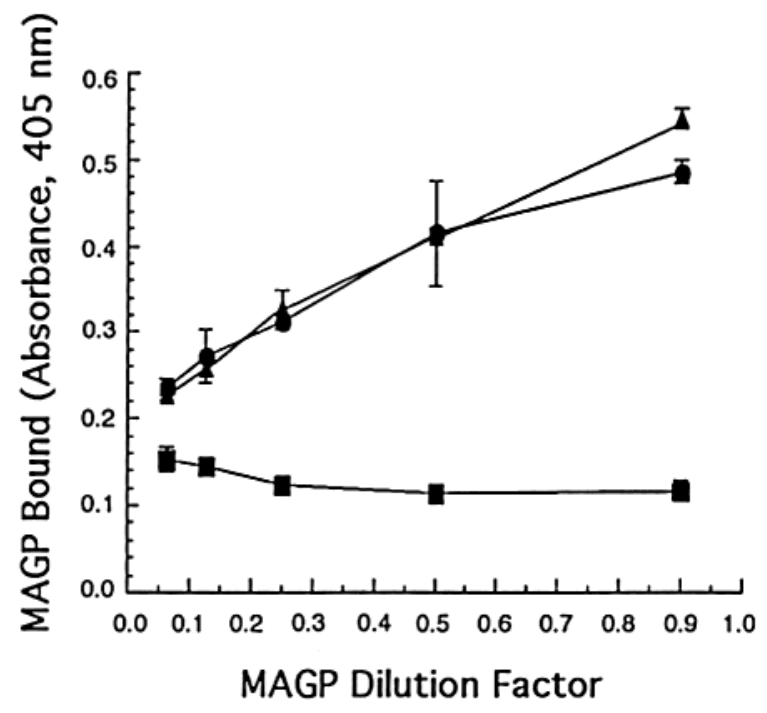

FIGURE 9: Binding to tropoelastin does not require sulfation of MAGP-1. (A) Immunoprecipitation of MAGP-1 from medium of untreated (lanes 1 and 2) or chlorate-treated (lanes 3 and 4) MAGP1-expressing CHO cells. Lanes 1 and 4 are from cells labeled with $\left[{ }^{35} \mathrm{~S}\right]$-cysteine and lanes 2 and 3 are from cells labeled with $\left[{ }^{35} \mathrm{~S}\right]-$ sulfate. Lanes 1 and 4 demonstrate that chlorate treatment had no effect on the synthesis of MAGP-1 or its ability to be recognized by the MAGP-1 antibody. Lane 3 confirms that the sulfation of MAGP-1 was inhibited in the presence of chlorate. (B) Medium from untreated or chlorate-treated MAGP-1-expressing $\mathrm{CHO}$ cells was added to ELISA plates coated with full-length recombinant tropoelastin. MAGP-1 bound to tropoelastin was quantified using the MAGP-1 antibody and a colorimetric assay. MAGP-1 from untreated (circles) and chlorate-treated (triangles) cells demonstrated equivalent binding to tropoelastin. Medium from untransfected $\mathrm{CHO}$ cells served as a control (squares).

aggregation. The ability of the glutamine-containing peptide to form insoluble fibrils that demonstrated green birefringence after congo red staining indicates that the peptide aggregates have $\beta$-pleated sheet structural features in common with amyloid-like fibrils $(25,26)$. Indeed, Aggeli et al. (27) have shown that synthetic peptides with glutamineenriched sequences similar to the sequence found in MAGP-1 produce $\beta$-sheet structures that self-associate to form insoluble gels. The significance of MAGP-1 self-association through the glutamine-rich region is not known, but it may have some physiological role in the organization of this protein into homo- or heteropolymers within the extracellular matrix. It may also suggest a mechanism whereby MAGP-1 could interact with other molecules capable of forming $\beta$-sheet structures.

Throughout this and other studies (2), we have noticed that aggregation of full-length MAGP-1 occurs much more slowly and is much less complete than what is observed for the front half construct. One explanation for this difference in aggregation is that the Gln-rich region in the full-length protein may not be as accessible for protein-protein interactions as it is in the front half construct alone. This raises the interesting possibility that conformational changes induced by proteolysis or by binding of MAGP-1 to other proteins may convert the sequence into an active conformation.

MAGP-1 was initially described as a glycoprotein based on its reactivity with PAS reagent and the detection by sugar analysis of associated $\mathrm{N}$-acetylgalactosamine, galactose, mannose, and glucose carbohydrates (14). When the fulllength cDNA for MAGP-1 was characterized and no Nlinked glycosylation consensus sequences were found, it was suggested that carbohydrates must be O-linked (17). Our finding that MAGP-1 secreted by $\mathrm{CHO}$ cells incorporates radiolabeled carbohydrate and interacts specifically with lectins confirms that the protein undergoes O-linked glycosylation. It is important to note that the extent of MAGP-1 glycosylation is not extensive since the apparent molecular weight of the fully glycosylated protein (either the full-length or front half construct) does not differ significantly from the nonglycosylated form. This suggests that the carbohydrates linked to MAGP-1 are the small tri- and tetrasaccharide varieties and not the larger mucin-like chains (28).

The precise sites of glycosylation within the molecule remain elusive. Because the specificity of the transferase that links the carbohydrate GalNAc to the serine or threonine side chain is presently unknown, the prediction of O-linked glycosylation sequences is still inaccurate. Our studies have localized carbohydrate incorporation to the front half of the protein. However, the capacity of single glycosylation site mutants to bind lectins indicates that MAGP-1 contains more than one site of glycosylation. The biological function of MAGP-1 carbohydrate modification is still unknown, although glycosylation of other proteins has been shown to regulate protein - protein and protein-cell interactions (29).

The presence of sulfate moieties within the MAGP-1 molecule were first demonstrated by Tomasini-Johansson et al. (30) who noted that a 30-kDa sulfated protein, identified as MAGP-1 by antibody reactivity, interacted with a monoclonal antibody raised against human vitronectin. The antibody did not react with protein isolated from cells treated with chlorate to inhibit sulfation, suggesting that the antibody recognized a sulfate moiety on both molecules. In addition, sulfation of MAGP-1 was suggested to be on tyrosine residue(s) since digestion with chondroitinase did not affect the level of sulfation, confirming that MAGP-1 is not a proteoglycan. Our studies confirm that sulfation of MAGP-1 is occurring within a consensus sequence for tyrosine sulfation (Y 47, 48, and 50) that is homologous to that present in vitronectin (30).

The role of MAGP-1 sulfation in elastic matrix assembly or function is unknown, but the importance of the modification was suggested by our studies showing that inhibition of sulfation prevents microfibrillar protein incorporation into the ECM of cultured cells (31). Our direct binding studies show that sulfation of MAGP-1 is not required for its 
interaction with tropoelastin. We cannot exclude the possibility, however, that sulfation might alter the affinity of the interaction.

In addition to containing sites for sulfation and glycosylation, the amino-terminal region of MAGP-1 contains an amine acceptor site for tissue transglutaminase. Our previous studies with full-length MAGP-1 suggested that a transglutaminase site was located near the amino-terminus (2), but the precise residues involved in the reaction were not determined. Results in Figure 3 show that a small aminoterminal fragment of MAGP-1 containing the first four glutamine residues (Q18, Q20, Q34, and Q41) serves as an amine-acceptor. On the basis of the preference of transglutaminase for modifying the second glutamine residue in a Q-X-Q pair, Q20 was the most likely target residue. Indeed, the mutation of Q20 prevents modification by this crosslinking enzyme, verifying that this residue can serve as a substrate for transglutaminase. Mutation of Q34 resulted in a significant decrease in $\left[{ }^{14} \mathrm{C}\right]$-spermidine incorporation, suggesting that this site could influence the transglutaminase reaction at residue $\mathrm{Q} 20$, or that it might be a second transglutaminase site. It should be noted that peptide fragments downstream of residue 58 were not studied and we cannot exclude other transglutaminase sites in this region of the molecule.

In an earlier study, we speculated that the high molecular weight aggregates observed when MAGP-1 was produced at high levels in a baculoviral expression system arose as the result of transglutaminase-mediate cross-linking. Our current results, however, suggest that aggregation occurs through $\beta$-sheetlike associations mediated by the polyglutamine sequence in the molecule. While transglutaminase cross-linking may stabilize these polymeric structures, it is also possible that cross-links between MAGP-1 and other molecules of the microfibril, such as fibrillin or tropoelastin, may be important in stabilizing microfibrillar structure. Indeed, like MAGP-1, both fibrillin and tropoelastin have been shown to be substrates for transglutaminase $(3,32)$.

As with most proteins of the extracellular matrix, posttranslational modifications add diversity and specificity to biological function. Characterizing the modifications that occur on MAGP-1 has been particularly difficult because of the protein's unusual physical properties. The expression of the recombinant MAGP-1 proteins described in this report has allowed us to more finely map many of the modifications that occur. These results are important in understanding the macromolecular interactions, and therefore the function, of MAGP-1.

\section{REFERENCES}

1. Ross, R., and Bornstein, P. (1969) J. Cell Biol. 40, 366-381.

2. Brown-Augsburger, P., Broekelmann, T., Mecham, L., Mercer, R., Gibson, M. A., Cleary, E. G., Abrams, W. R., Rosenbloom, J., and Mecham, R. P. (1994) J. Biol. Chem. 269, 2844328449.

3. Qian, R. Q., and Glanville, R. W. (1997) Biochemistry 36, $15841-15847$.
4. Sakai, L. Y., Keene, D. R., and Engvall, E. (1986) J. Cell Biol. 103, 2499-2509.

5. Zhang, H., Adfelroth, S. D., Hu, W., Davis, E. C., Sanguineti, C., Bonadio, J., Mecham, R. P., and Ramirez, F. (1994) J. Cell Biol. 124, 855-863.

6. Reinhardt, D. P., Keene, D. R., Corson, G. M., Poschl, E., Bachinger, H. P., and Gambee, J. E. (1996) J. Mol. Biol. 258, 104-116.

7. Gibson, M. A., Hatzinikolas, G., Kumaratilake, J. S., Sandberg, L. B., Nicholl, J. K., Sutherland, G. R., and Cleary, E. G. (1996) J. Biol. Chem. 271, 1096-1103.

8. Henderson, M., Polewski, R., Fanning, J. C., and Gibson, M. A. (1996) J. Histochem. Cytochem. 44, 1389-1397.

9. Davis, E. C. (1994) J. Cell Sci. 107, 727-736.

10. Kumaratilake, J. S., Gibson, M. A., Fanning, J. C., and Cleary, E. G. (1989) Eur. J. Cell Biol. 50, 117-127.

11. Gibson, M. A., Finnis, M. L., Kumaratilake, J. S., and Cleary, E. G. (1998) J. Histochem. Cytochem. 46, 871-885.

12. Brown-Augsburger, P. B., Broekelmann, T., Rosenbloom, J., and Mecham, R. P. (1996) Biochem. J. 318, 149-155.

13. Finnis, M. L., and Gibson, M. A. (1997) J. Biol. Chem. 272, $22817-22823$.

14. Gibson, M. A., Hughes, J. L., Fanning, J. C., and Cleary, E. G. (1986) J. Biol. Chem. 261, 11429-11436.

15. Mecham, R. P. (1987) Methods Enzymol. 144 (D), 232-246.

16. Sambrook, J., Fritsch, E., and Maniatis, T. (1989) Molecular Cloning: A Laboratory Manual, 2nd ed., Cold Spring Harbor Laboratory, Cold Spring Harbor, NY.

17. Gibson, M. A., Sandberg, L. B., Grosso, L. E., and Cleary, E. G. (1991) J. Biol. Chem. 266, 7596-7601.

18. Ausubel, F., Brent, R., Kingston, R., Moore, D., Seidman, J., Smith, J., and Struhl, K. (1992) in Current Protocols in Molecular Biology, Chapter 16, Section 14, John Wiley and Sons, New York.

19. Caputo, C. B., Fraser, P. E., Sobel, I. E., and Kirschner, D. A. (1992) Arch. Biochem. Biophys. 292, 199-205.

20. Hansen, J. E., Lund, O., Rapacki, K., and Brunak, S. (1997) Nucleic Acids Res. 25, 278-282.

21. Hansen, J. E., Lund, O., Tolstrup, N., Gooley, A. A., Williams, K. L., and Brunak, S. (1998) Glycoconjugate J. 15, 115-130.

22. Bashir, M. M., Abrams, W. R., Rosenbloom, J., Kucich, U., Bacarra, M., Han, M.-D., Brown-Augsburger, P., Mecham, R. P., and Rosenbloom, J. (1994) Biochemistry 33, 593-600.

23. Huttner, W. B., and Baeuerle, P. A. (1988) Mol. Cell Biol. 6, 97-140.

24. Baeuerle, P. A., and Huttner, W. B. (1986) Biochem. Biophys. Res. Commun. 141, 870-877.

25. Scherzinger, E., Lurz, R., Turmaine, M., Mangiarini, L., Hollenbach, B., Hasenbank, R., Bates, G. P., Davies, S. W., Lehrach, H., and Wanker, E. E. (1997) Cell 90, 549-558.

26. DePace, A. H., Santoso, A., Hillner, P., and Weissman, J. S. (1998) Cell 93, 1241-1252.

27. Aggeli, A., Bell, M., Boden, N., Keen, J. N., Knowles, P. F., McLeish, T. C., Pitkeathly, M., and Radford, S. E. (1997) Nature 386, 259-262.

28. Stanley, P. (1993) in Cell Surface and Extracellular Glycoconjugates (Roberts, D. D., and Mecham, R. P., Eds.) pp 181222, Academic Press, San Diego.

29. Varki, A. (1993) Glycobiology 3, 97-130.

30. Tomasini-Johansson, B. R., Ruoslahti, E., and Pierschbacher, M. D. (1993) Matrix 13, 203-214.

31. Trask, B. C., Trask, T. M., Broekelmann, T., and Mecham, R. P. (2000) Mol. Biol. Cell 11, 1499-1507.

32. Mecham, R. P., Broekelmann, T., Davis, E. C., Gibson, M. A., and Brown-Augsburger, P. (1995) Ciba Foundation Symposium 192, 172-180.

BI002738Z 